\title{
Molecular diversity of protozoa in rumen of Indian buffalo (Bubalus bubalis)
}

\author{
K. M. Singh · P. R. Pandya $\cdot$ A. K. Tripathi $\cdot$ G. R. Patel $\cdot$ S. Parnerkar • \\ R. K. Kothari • C. G. Joshi
}

Received: 4 August 2012/ Accepted: 17 July 2013/Published online: 29 August 2013

(C) NAAS (National Academy of Agricultural Sciences) 2013

\begin{abstract}
Protozoa communities in buffalo rumen were characterized using 18S rRNA gene library prepared from the pooled DNA sample obtained from three adult animals. A total of 172 clones were sequenced, which were grouped into 53 operational taxonomic units (OTUs) based on unique 18S r DNA sequences with $95 \%$ confidence intervals. Phylogenetic analyses showed that 40 OTUs (124 of 172 clones) belonged to uncultured protozoa group, indicating that this group is most dominant component of protozoa resident in rumen of Indian buffalo. 44 clones (12 OTUs) belonged to the class Kinetofragminophorea. Among Kinetofragminophorea, 44 clones fell into two species identified as Dasytricha ruminantium-like clone (27 clones) and Isotricha prostoma-like clone (17 clones). These include 11 single-clone OTUs, so Good's coverage $(93.75 \%)$ of $18 \mathrm{~S}$ rRNA libraries indicated that the sequences identified in the libraries represent the majority of protozoa diversity present in rumen.
\end{abstract}

Keywords Buffalo rumen $\cdot$ Protozoa $\cdot 18 \mathrm{~S}$ rDNA $\cdot$ Phylogenetic analysis

\section{Introduction}

Protozoa are unicellular eukaryotic microorganisms which are ubiquitous in nature and anthropogenic environments. The rumen ciliates are potentially an agriculturally important group of protozoa found in domestic and wild ruminants [30]. Several factors seem to influence the

K. M. Singh $(\varangle) \cdot$ A. K. Tripathi · C. G. Joshi

Department of Animal Biotechnology, College of Veterinary Science and Animal Husbandry, Anand Agricultural University, Anand 388 001, Gujarat, India

e-mail: kmsingh18@gmail.com

P. R. Pandya · G. R. Patel · S. Parnerkar

Animal Nutrition Research Station, AAU, Anand, Gujarat, India

R. K. Kothari

Department of Microbiology, Christ College, Rajkot, Gujarat, India

K. M. Singh

Department of Genetics, ARIBAS, New V V Nagar, Anand, India composition of the protozoan population in the rumen. These include type and amount of feed consumed, $\mathrm{pH}$, turnover rate, and feed level. The rumen microorganisms utilize carbohydrates as a carbon source in energy-yielding processes, whereas hydrogen gas $\left(\mathrm{H}_{2}\right)$ is released as byproduct during ATP generation. In the protozoa, in turn, benefit from hydrogen removal by the methanogens because hydrogen is inhibitory to protozoan metabolism [32]. Earlier reports have provided evidence of strong relationships, such as endosymbiosis between ruminal protozoa and methanogens $[8,28]$. Ciliate protozoa play a diverse role in the ruminal metabolism of nutrients. To improve the efficiency of feed crude protein utilization, considerable effort has been made to find a means of total elimination of protozoa from the rumen (defaunation) and a massive reduction in the rumen protozoan population (reduced fauna). Chemical drenching of experimental animals has been found to improve milk production. Detection and identification of protozoa have commonly been achieved through microscopic examination of morphological features. It remains difficult and time-consuming to reliably detect or identify many protozoan species by these 
methods, as protozoa may be fragile and inconspicuous and as it may be difficult to determine whether a given morphological feature can be regarded as distinct or not $[3,9$, 23]. The anaerobic ruminal protozoa have been well studied [31], but much of this work is based on microscopic examination [4]. Difficulties in cultivating protozoa, and their polymorphic nature, have delayed effective assessment of protozoan ecology and taxonomy [5]. The small subunit ribosomal RNA (SSU-rRNA) gene called $16 \mathrm{~S}$ rRNA in prokaryotes and $18 \mathrm{~S}$ rRNA in eukaryotes is widely used as molecular marker to identify morphologically indistinguishable species, to infer their phylogenetic relationships, and to elucidate diversity. PCR-sequencing methods have been extensively used to examine the protozoal diversity in rumen samples [13]. PCR-DGGE has also been used in the profiling of protozoal communities in the rumen $[20,24]$.

India possesses more than $50 \%$ of world's buffalo population; Indian buffalo produce more than $50 \%$ milk in India [15]. Surti is a popular breed of buffalo found in central Gujarat state. The Surti buffaloes are of medium size having docile temperament and body weight $300-350 \mathrm{~kg}$ at maturity. Since our animals mainly sustain on crop residues, the protozoan population is expected much different than that of exotic cattle. The present study was conducted to examine the diversity of rumen protozoa in Surti buffalo offered diet, green fodder bajra (Pennisetum purpureum), mature pasture grass (Dichanthium annulatum), and compound concentrate mixture. The molecular techniques were used to construct a library of $18 \mathrm{~S}$ rDNA clones of rumen protozoa, and a phylogenetic tree for the clones isolated.

\section{Materials and Methods}

\section{Sampling and DNA Extraction}

The permission of the Committee for the Purpose of Control and Supervision of Experiments on Animals (CPCSEA) was obtained prior to initiation of the study. The experiments were carried out on three adult Surti buffaloes (Bubalus bubalis), approximately 3 years of age with a mean live weight of $201 \pm 18 \mathrm{~kg}$, which were reared at the Department of Animal Nutrition, College of Veterinary Science and A.H. Anand. All the animals were maintained under uniform feeding regime (ICAR 1998) for minimum 21 days for dietary adaptation. The diet comprised of green fodder bajra ( $P$. purpureum), mature pasture grass (D. annulatum), and compound concentrate mixture $(20 \%$ CP, $65 \%$ TDN). The animals were offered $10 \mathrm{~kg}$ green, ad-lib dry grass and $2.5 \mathrm{~kg}$ of concentrate mixture daily. Animals were let loose daily for $2 \mathrm{~h}$ in the morning and evening during which they had free access to drinking water. Approximately $500 \mathrm{ml}$ of rumen fluid was collected after 21 days (dietary adaptation) using flexible tube at $0,2,4$, and $6 \mathrm{~h}$ after feeding [14]. About $100 \mathrm{ml}$ rumen fluid was passed through four layers of cheese cloth (autoclave) in laminar air flow to remove particulate matter. Remaining rumen fluid was stored at $-80{ }^{\circ} \mathrm{C}$ for further study. Total DNA $(0,2,4,6 \mathrm{~h} \times 3$ animals $)$ were extracted from pooled sample by using a commercially available kit according to the manufacturer's instructions (QIAGEN Stool kit; QIAGEN, CA). The total DNA mixture was used as a template in PCR to amplify $18 \mathrm{~S} r$ DNA.

\section{PCR Primers and Amplification}

A protozoa-specific forward primer (5'-ACTTTCGATGGTAGTGTATTGGACTAC-3') was used with a Eukarya-specific reverse primer (5'-ATGATCCTTCTGCAGGTTCACCTAC-3') [19]. Subsequently, 18S r DNA fragment were amplified by PCR using the metagenomic DNA. A total of $25 \mu \mathrm{l}$ of reaction mixture consisted of $10 \mathrm{pmol}$ of each primer, $30 \mathrm{ng}$ of template DNA, and $12.5 \mu \mathrm{l}$ of master mix (Fermentas, UK). The PCR amplification was performed by Thermal Cycler (ABI, USA) and the PCR conditions were as follows: initial denaturation at $95{ }^{\circ} \mathrm{C}$ for $5 \mathrm{~min}, 30$ cycles of amplification consisting of a 1-min denaturation step at $94{ }^{\circ} \mathrm{C}$, a 1-min annealing step at $37{ }^{\circ} \mathrm{C}$, a 3-min extension step at $72{ }^{\circ} \mathrm{C}$, and a final 10-min extension at $72{ }^{\circ} \mathrm{C}$. The anticipated product of approximately $1.36 \mathrm{~kb}$ was cleaned using a Qiagen DNA Gel Extraction Kits (QIAGEN, CA) in accordance with the directions of the manufacturer.

\section{Cloning and Sequencing}

The purified PCR products were cloned in InstaT/A cloning kit (Fermentas, UK) as per the instructions of the manufacturer. The recombinant plasmids after were extracted by the Qiagen mini-prep plasmids extraction kit (QIAGEN, CA). Plasmid inserts were amplified with primers $\mathrm{M} 13 \mathrm{~F}$ (5'-GTAAAACGACGGCCAG-3') and M13R (5'-CAGGAAACAGCTATGAC- $3^{\prime}$ ) and nucleotide sequences of cloned genes were determined by sequencing with $\mathrm{M} 13 \mathrm{~F} /$ M13R primer in ABI Prism 310 Genetic analyser (Applied Biosystems Inc., CA) using BigDye Terminator (version 3.1) at the Animal Biotechnology Laboratory, AAU, Anand, Gujarat, India. Finally a total of 172 clones were sequenced.

Sequence Analysis

All reference sequences were obtained from the GenBank/ EMBL/DDBJ/ [2]. Sequences ( 550 bp) from the current 
study were analysed by the CHECK_CHIMERA program [18] to remove any chimera rDNA clone. The similarity searches for sequences were carried out by BLAST (http://www.ncbi.nlm.nih.gov/ BLAST/Blast.cgi [17] and alignment was done using CLUSTAL W (http://www. ebi.ac.uk/Tools/clustalw2/index.html [26]. Ambiguously and incorrectly aligned positions were aligned manually. The distance matrix was calculated using the DNADIST program included in PHYLIP [7] and used to assign sequences in various operational taxonomic units (OTUs) or phylotypes by DOTUR [21] with $95 \%$ confidence intervals to quantify the diversity of phylotypes and total of 53 OTUs were distinguished, based on unique 18S rDNA sequences. The percentage of Good's coverage was calculated as $[1-(n / N)] \times 100$, where ' $n$ ' is the number of single-clone OTUs and ' $N$ ' is the library size. Phylogenetic tree was constructed by the neighbor-joining method using MEGA 4.0 [25]. Bootstrap re-sampling analysis for 1,000 replicates was performed to estimate the confidence of tree topologies [7].

Nomenclature and Nucleotide Sequence Accession Numbers

The prefix IBRP was used to denote OTU identified and nucleotide sequences have been deposited in the GenBank database under the accession numbers EU345005 to EU345176.

\section{Results}

Sequence Analysis

All the sequences (172) were subjected to online homology search in GenBank [2] which implements the BLAST algorithm [17]. The summary of Blast result are given in Table 1. The sequences generated in this study showed 79-96 \% sequence similarities with the sequences of protozoa available in the GenBank. The clones sequenced in this study were grouped into 53 OTUs, based on rDNA sequences. Of the 53 OTUs, 12 had $>90 \%$ similarity with the $18 \mathrm{~S}$ rDNA sequences available in the GenBank, 27 had $86-90 \%$ similarity and 14 had $<85 \%$ similarity.

\section{Phylogenetic Analysis}

The phylogenetic analysis of the sequences (Fig. 1) showed that 40 OTUs, representing 124 clones, belonged to unidentified protozoa. Twelve OTUs (44 clones) belonged to the class Kinetofragminophorea. Within the Kinetofragmi nophorea, seven OTUs, representating 27 clones, grouped with Dasytricha ruminantium-like clones and five OYUs representing 17 clones grouped with Isotricha prostoma-like clones. The phylogenetic analysis indicate that the OTU19 (4 Clones) belonged to haptorida. However, Blast results hit with known protozoa i.e. Troglodytella abrassarti. This is due to very low similarity (Table 2). Thus, 18S rRNA sequences obtained from rumen formed tightly-clustered affiliated to the different groups. The total 11 single-clone OTUs, so Good's coverage $(93.75 \%)$ of $18 \mathrm{~S}$ rDNA libraries indicated that the sequences identified in libraries represent the protozoan diversity in the rumen.

\section{Discussion}

The molecular inventory of protozoa revealed in present study showed the occurrence of complex protozoa communities in buffalo rumen ecosystem. The number and distribution of phylotypes indicates the protozoa diversity in rumen of Surti buffalo. Compared to other ecosystems, there is no previous information about the rumen protozoa of Indian buffalo. The relative lack of information on ruminal protozoa may be due to difficulties with isolation, culture, or maintenance. Rumen isolates often lose viability for unknown reasons during purification or sub culturing of pure isolates. More than 24 genera of ruminal protozoa have been described based on cultivation and morphological studies [4, 29]). Although, sequences of few genera are available in sequence databases. Most genera are representatives of typical bovine rumen populations viz Entodinium, Diplodinium, Eudiplodinium, Ostracodinium, Metadinium, Enoploplastron, Polyplastron, Epidinium, Ophryoscolex, Isotricha, and Dasytricha [13, 30]. The present study revealed the phylogenetic diversity of the protozoan community in the rumen fluid of Surti buffalo by analyzing protozoan $18 \mathrm{~S}$ rDNA sequences. BLASTn searches showed that sequenced clones shared similarity (79-96 \%) with ruminal protozoan sequences with GenBank database. The rumen fluid library was classified into three phylogenetic groups. The largest group was affiliated with the unidentified protozoa (40 OTUs, 124 clones), second group affiliated with the Kinetofragminophorea protozoa (12 OTUs, 44 clones), and third the group affiliated with the Haptorida protozoa (01 OTU, 04 clones). The predominant protozoa identified in this study were the unidentified group (Table 2). These results agree with the report of Isotricha and Dasytricha genera in the cattle rumen $[13,16]$. High number of Entodinium sp. has been reported by Akbar et al. [1] in Ghizel Sheep fed in pasture and nourished by dried grape by-product and Leng et al. [16] in Yunnan Yellow Cattle fed malt meal. Interestingly, 
Table 1 Similarity values of $18 \mathrm{~S}$ rRNA sequences retrieved from the rumen fluid of Surti buffaloes

\begin{tabular}{|c|c|c|c|c|}
\hline OTU & No. of clone & Nearest relative & Accession no. & Similarity $(\%)$ \\
\hline IBRP1 & 02 & Ostracodinium gracile & AM158468 & 87 \\
\hline IBRP2 & 04 & Uncultured rumen protozoa & AF502929 & 89 \\
\hline IBRP3 & 03 & Ostracodinium gracile & AM158468 & 89 \\
\hline IBRP4 & 12 & Uncultured Canadian Arcott wether rumen protozoa & DQ832560 & 88 \\
\hline IBRP5 & 04 & Ostracodinium gracile & AM158468 & 90 \\
\hline IBRP6 & 04 & Uncultured Canadian Arcott wether rumen protozoa & DQ832560 & 87 \\
\hline IBRP7 & 03 & Cycloposthium ishikawai & EF632076 & 86 \\
\hline IBRP8 & 04 & Cycloposthium edentatum & EF632077 & 86 \\
\hline IBRP9 & 06 & Uncultured rumen protozoa clone YCRPB55 & EU163779 & 87 \\
\hline IBRP10 & 03 & Isotricha prostoma & AM158455 & 88 \\
\hline IBRP11 & 05 & Uncultured Canadian Arcott wether rumen protozoa & DQ832560 & 94 \\
\hline IBRP12 & 06 & Uncultured Canadian Arcott wether rumen protozoa & DQ832564 & 92 \\
\hline IBRP13 & 01 & Uncultured ciliate & AM158846 & 86 \\
\hline IBRP14 & 03 & Ostracodinium gracile & AM158468 & 88 \\
\hline IBRP15 & 05 & Isotricha prostoma & AM158455 & 92 \\
\hline IBRP16 & 02 & Uncultured rumen protozoa & EU163783 & 81 \\
\hline IBRP17 & 02 & Ostracodinium gracile & AM158468 & 89 \\
\hline IBRP18 & 10 & Isotricha prostoma & AM158455 & 91 \\
\hline IBRP19 & 04 & Troglodytella abrassarti & AB437347 & 86 \\
\hline IBRP20 & 02 & Uncultured Canadian Arcott wether rumen protozoa & DQ832565 & 84 \\
\hline IBRP21 & 03 & Uncultured rumen protozoa clone CRA9 & AF502927 & 86 \\
\hline IBRP22 & 06 & Teuthophrys trisulca africana & DQ411863 & 85 \\
\hline IBRP23 & 08 & Uncultured rumen protozoa clone YCRPB55 & EU163779 & 89 \\
\hline IBRP24 & 11 & Dasytricha ruminantium & AM158463 & 91 \\
\hline IBRP25 & 02 & Uncultured rumen protozoa & EU163779 & 84 \\
\hline IBRP26 & 02 & Uncultured rumen protozoa clone CRA5 & AF502923 & 83 \\
\hline IBRP27 & 06 & Uncultured rumen protozoa clone YCRPB55 & EU163779 & 89 \\
\hline IBRP28 & 02 & Uncultured ciliate & AM158846 & 89 \\
\hline IBRP29 & 02 & Uncultured ciliate & AM158873 & 91 \\
\hline IBRP30 & 07 & Dasytricha ruminantium & AM158463 & 86 \\
\hline IBRP31 & 02 & Ostracodinium gracile & AM158468 & 87 \\
\hline IBRP32 & 04 & Ostracodinium gracile & AM158468 & 89 \\
\hline IBRP33 & 01 & Isotricha prostoma & AM158454 & 84 \\
\hline IBRP34 & 02 & Uncultured rumen protozoa clone YCRPB59 & EU163783 & 85 \\
\hline IBRP35 & 04 & Dasytricha ruminantium & AM158463 & 87 \\
\hline IBRP36 & 01 & Uncultured rumen protozoa & EU163779 & 93 \\
\hline IBRP37 & 01 & Uncultured rumen protozoa clone YCRPB55 & EU163779 & 86 \\
\hline IBRP38 & 02 & Uncultured rumen protozoa clone YCRPB55 & EU163779 & 91 \\
\hline IBRP39 & 01 & Polyplastron multivesiculatum & AM158458 & 92 \\
\hline IBRP40 & 03 & Uncultured rumen protozoa clone YCRPB59 & EU163783 & 91 \\
\hline IBRP41 & 01 & Ostracodinium gracile & AM158468 & 89 \\
\hline IBRP42 & 02 & Uncultured Canadian Arcott wether rumen protozoa & DQ832560 & 83 \\
\hline IBRP43 & 01 & Dasytricha ruminantium & AM158463 & 86 \\
\hline IBRP44 & 01 & Isotricha prostoma & AM158456 & 84 \\
\hline IBRP45 & 02 & Cycloposthium ishikawai & EF632076 & 89 \\
\hline IBRP46 & 02 & Uncultured rumen protozoa clone YCRPB59 & EU163783 & 82 \\
\hline IBRP47 & 01 & Uncultured rumen protozoa clone YCRPB1 & EU163725 & 91 \\
\hline IBRP48 & 02 & Uncultured ciliate & AM158846 & 96 \\
\hline
\end{tabular}


Table 1 continued

\begin{tabular}{llllc}
\hline OTU & No. of clone & Nearest relative & Accession no. & Similarity (\%) \\
\hline IBRP49 & 01 & Ostracodinium gracile & AM158468 & 80 \\
IBRP50 & 01 & Uncultured rumen protozoa clone YCRPB65 & EU163789 \\
IBRP51 & 01 & Isotricha prostoma & AM158454 & 86 \\
IBRP52 & 01 & Ostracodinium gracile & AM158468 & 80 \\
IBRP53 & 01 & Uncultured rumen protozoa clone YCRPB59 & EU163783 & 82 \\
\hline
\end{tabular}

Total $=172$ clones

Fig. 1 Phylogenetic relationships of partial $18 \mathrm{~S}$ rRNA sequences of clones recovered from Surti buffalo rumen samples. The rooted tree was inferred by the neighborjoining method with 1,000 bootstrap replicates using the MEGA 4 tree building program. The Paramecium tetraurelia (X03772) are used as the outgroup for rooting the tree. The scale bar represents $5 \%$ sequence divergence. The symbol filled square indicates the reference sequences, open circle indicates Dasytricha ruminantium-like clone, filled circle indicates Isotricha prostoma-like clone, open square indicates haptorida protozoa, and filled triangle indicates unidentified protozoa; reference sequence (AB437347:

Troglodytella abrassarti)

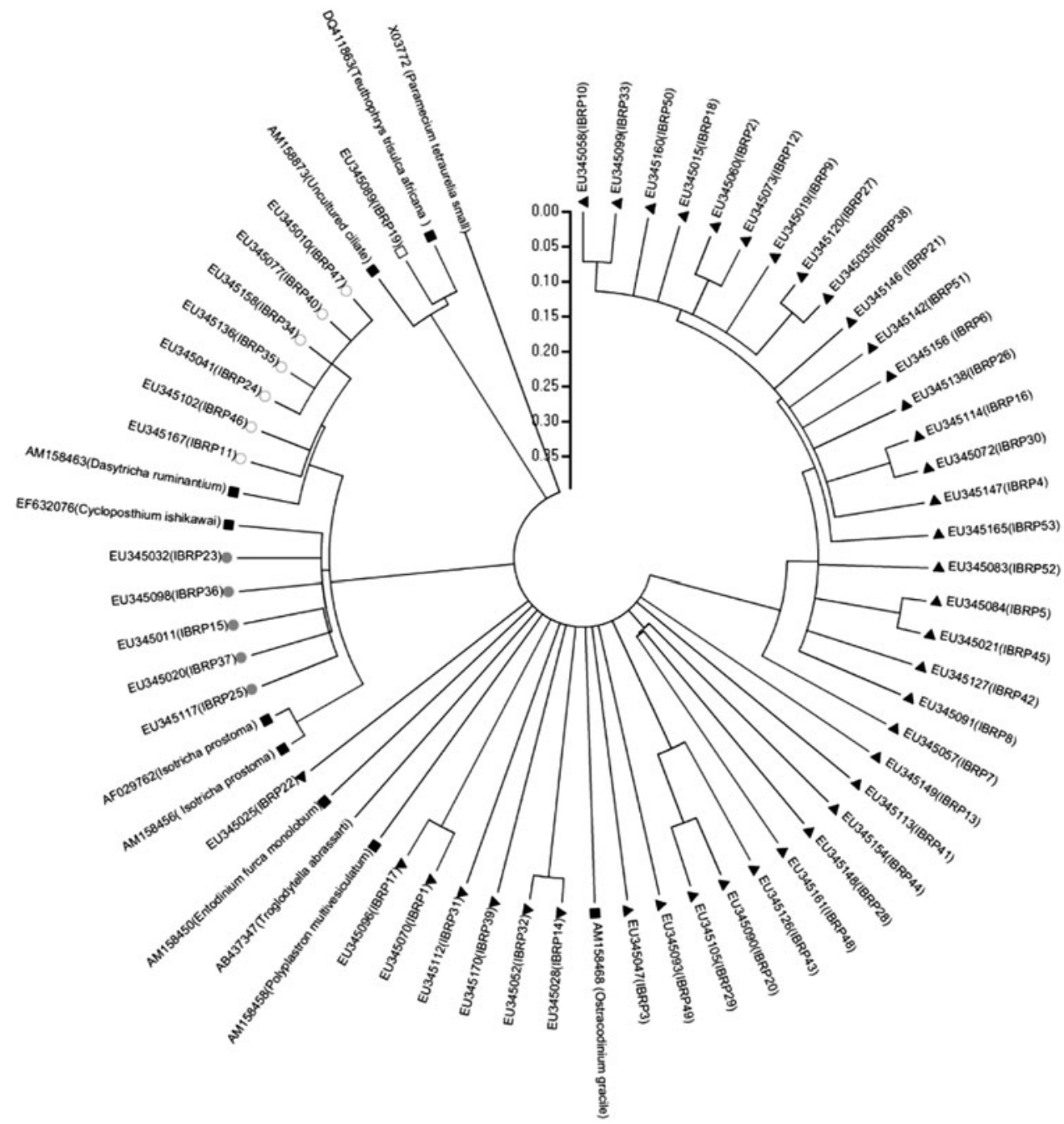

we could not detect the sequences related to Entodinium sp.; this may be due to the diet composition. Dehority and Odenyo [6] suggest that a selection for highly concentrated feed stuffs would lead to an Entodinium only fauna, as they observed in Grant's gazelle. Karnati et al. [13] reported that their protozoan-specific primers (used here) had a single mismatch with the 18S rDNA of Entodinium sp., but matched exactly with the $18 \mathrm{~S}$ rDNA sequences of other protozoan species. They suggested that a PCR primer degenerates at the mismatched position would help minimize PCR bias, allowing for more representative retrieval of ruminal protozoan 18S rDNA from complex ruminal samples. The diversities of different clone libraries of rumen protozoa have been given in Table 3 .

However, Isotricha sp. and Polyplastron sp. were identified in the washed ciliate suspension and many 
Table 2 Analysis of $18 \mathrm{~S}$ rDNA phylotypes diversity retrieved from the rumen fluid of Surti buffaloes

\begin{tabular}{llc}
\hline Taxon & $\begin{array}{l}\text { No. of operational } \\
\text { taxonomic units } \\
\text { (OTUs) }\end{array}$ & No. of clones \\
\hline 1.Unidentified protozoa & 40 & 124 \\
2. Kinetofragminophorea & 12 & 44 \\
a. Dasytricha ruminantium-like clone & 07 & 27 \\
b. Isotricha prostoma-like clone & 05 & 17 \\
3. Haptorida protozoa & 01 & 04 \\
Total & 53 & 172 \\
\hline
\end{tabular}

Table 3 The diversity comparing of different clone libraries of rumen protozoa

\begin{tabular}{|c|c|c|c|c|c|c|c|}
\hline \multirow{2}{*}{$\begin{array}{l}\text { Clone libraries } \\
\text { Animals }\end{array}$} & \multicolumn{2}{|c|}{ Karnati et al. [13] } & \multirow{2}{*}{$\begin{array}{l}\text { Shin et al. [22] } \\
\text { Cow }\end{array}$} & \multicolumn{2}{|c|}{ Leng et al. [16] } & \multirow{2}{*}{$\begin{array}{l}\text { Fuente et al. [10] } \\
\text { Spanishi bex }\end{array}$} & \multirow{2}{*}{$\begin{array}{l}\text { Our library } \\
\text { Surti buffalc }\end{array}$} \\
\hline & Cow & & & Yunnan ye & attle & & \\
\hline Clone numbers & 12 & 11 & 37 & 66 & 55 & 8 & 172 \\
\hline Diets & Ration $(1: 1)^{\mathrm{b}}$ & Alfalfa & Ration $(1: 4)^{\mathrm{c}}$ & Malt meal & Straw & Pasture & Mix ration $^{\mathrm{d}}$ \\
\hline \multicolumn{8}{|c|}{ Clone distribution (\%) } \\
\hline Entodinium & 97.3 & 77.2 & 81.1 & 72.7 & 60.0 & $>90$ & ND \\
\hline Dipladenia $^{\mathrm{a}}$ & 1.7 & 4.6 & ND & ND & ND & ND & $>13.0$ \\
\hline Epidinium & 0.3 & $<0.5$ & 18.9 & ND & ND & ND & ND \\
\hline Ophryoscolex & ND & 0.9 & ND & ND & ND & ND & ND \\
\hline Isotricha & $<0.3$ & 6.0 & ND & 16.7 & ND & ND & $>13.0$ \\
\hline Dasytricha & 0.7 & 11.2 & ND & 10.6 & ND & ND & $>10.0$ \\
\hline
\end{tabular}

ND not detectable

a Subfamily, containing the genera Diplodinium, Eudiplodinium, Ostracodinium, Metadinium, Enoploplastron, and Polyplastron

b The diet contained $50 \%$ forage (mixture of corn silage and haylage) and $50 \%$ concentrate (corn grain and soybean meal) on a DM basis

c $4: 1$ rice hull to concentrated

d The diet contained: green fodder Napier bajra 21 (Pennisetum purpureum), mature pasture grass (Dichanthium annulatum), and concentrate mixture (20\% crude protein, $65 \%$ total digestible nutrients)

methanogens were detected on ciliate cells by F420 auto fluorescence [27]. Similar results were also observed in our studies. Biochemical differences between Isotricha and Dasytricha have been examined by [11, 12]. Dasytricha is more versatile than Isotricha, fermenting cellobiose, galactose, and maltose. Fermentation products from galactose were the same as those formed from glucose. From glucose, the holotrich produced lactic, acetic, and butyric acids, carbon dioxide, hydrogen, and traces of propionic acid [12]. A single OTU (IBRP19) located within the haptorida protozoa and may represent a dietary transient.

\section{Conclusions}

In conclusion, Surti buffalo rumen harbors protozoal community in the rumen that is composed of several genera including, Dasytricha, Isotricha, Ostracodinium, Polyplastron, Cycloposthium), Teuthophrys, and Troglodytella. While majority of the sequences were unidentified. An advanced set of protozoan-specific phylogenetic probes and quantitative real time PCR assay are needed to their distribution throughout rumen microbial communities. Future studies to understand the effects of varying rumen protozoa on different animal feeding habits, digestion and methanogens will also be important.

Acknowledgments Financial support provided by the Department of Biotechnology, Govt. of India, New Delhi is gratefully acknowledged.

\section{References}

1. Akbar T et al (2009) The study of diversity of ciliate protozoa in Ghizel sheep fed in pasture and nourished by dried grape byproduct. Am J Anim Vet Sci 4(2):37-41

2. Benson DA et al (2007) GenBank. Nucleic Acids Res 35:D1-D25

3. Caron DA et al (2004) The growing contribution of molecular biology and immunology to protistan ecology: molecular signatures as ecological tools. J Eukaryot Microbiol 51:38-48

4. Dehority BA (1993) Laboratory manual for classification and morphology of rumen ciliate protozoa. CRC Press, Boca Raton 
5. Dehority BA (1994) Rumen ciliate protozoa of the blue duiker (Cephalophus monticola), with observations on morphological variation lines within the species Entodinium dubardi. J Eukaryot Microbiol 41:103-111

6. Dehority BA, Odenyo AA (2003) Influence of diet on the rumen protozoal fauna of indigenous African wild ruminants. J Eukaryot Microbiol 50:220-223

7. Felsenstein J (1985) Confidence limits on phylogenies: an approach using the bootstrap. Evolution 39:783-791

8. Finlay BJ et al (1994) Some rumen ciliates have endosymbiotic methanogens. FEMS Microbiol Lett 117:57-162

9. Fried $J$ et al (2002) Improvement of ciliate identification and quantification: a new protocol for fluorescence in situ hybridization (FISH) in combination with silver stain techniques. Syst Appl Microbiol 25:555-571

10. Fuente $G$ et al (2009) Rumen protozoal diversity in the Spanish ibex (Capra pyrenaica hispanica) As compared with domestic goats (Capra hircus). Eur J Protistol 45:112-120

11. Gutierrez J (1955) Experiments on the culture and physiology of holotrichs from the bovine rumen. Biochem J 60(3):516-522

12. Howard BH (1959) The biochemistry of rumen protozoa: carbohydrate fermentation by Dasytricha and Isotricha. Biochem J 71(4):671-675

13. Karnati SKR et al (1994) Technical note: specific PCR amplification of protozoal 18S rDNA sequences from DNA extracted from ruminal samples of cows. J Anim Sci 81:812-815

14. Khamapa $S$ et al (2006) Effects of urea level and sodium dimalate in concentrate containing high cassava chip on ruminal fermentation efficiency, microbial protein synthesis in lactating dairy cows raised under tropical condition. Asian Aust J Anim Sci 19:837-844

15. Kumar S et al (2007) Phylogeography and domestication of Indian river buffalo. BMC Evol Biol 7:186

16. Leng $J$ et al (2011) Assessment of protozoa in Yunnan Yellow Cattle rumen based on the 18S rRNA sequences. Mol Biol Rep 38(1):577-585

17. Madden TL et al (1996) Application of network BLAST server. Methods Enzymol 266:131-141

18. Maidak BL et al (2001) The RDP-II (Ribosomal Database Project). Nucleic Acids Res 29:173-174
19. Medlin L et al (1998) The characterization of enzymatically amplified eukaryotic 16S-like rRNA-coding regions. Gene 1:491-499

20. Regensbogenova M et al (2004) A re-appraisal of the diversity of the methanogens associated with the rumen ciliates. FEMS Microbiol Lett 238:307

21. Schloss PD, Handelsman J (2005) Introducing DOTUR, a computer program for defining operational taxonomic units and estimating species richness. Appl Environ Microbiol 71:1501-1506

22. Shin EC et al (2004) Phylogenetic analysis of protozoa in the rumen contents of cow based on the $18 \mathrm{~S}$ rDNA sequences. J Appl Microbiol 97:378-383

23. Sims GP et al (2002) Identification and phylogenetic analysis of morphologically similar naked amoebae using small subunit ribosomal RNA. J Eukaryot Microbiol 49:478-484

24. Sylvester JT et al (2005) Evaluation of a real-time PCR assay quantifying the ruminal pool size and duodenal flow of protozoal nitrogen. J Dairy Sci 88:2083-2095

25. Tamura K et al (2007) MEGA4: Molecular Evolutionary Genetics Analysis software version 4.0. Mol Biol Evol 24:1596-1599

26. Thompson JD et al (1994) CLUSTAL W: improving the sensitivity of progressive multiple sequence alignment through sequence weighting, position-specific gap penalties and weight matrix choice. Nucleic Acids Res 22:4673-4680

27. Tokura $M$ et al (1999) Phylogenetic study of methanogens associated with rumen ciliates. Curr Microbiol 39:123-128

28. Ushida K et al (1997) Ciliate protozoa and ruminal methanogenesis. In: Onodera R, Itabashi H, Ushida K, Yano H, Sasaki Y (eds) Rumen microbes and digestive physiology in ruminants. Japan Sci Soc Press, Tokyo, pp 209-220

29. Williams AG (1986) Rumen holotrich ciliate protozoa. Microbiol Rev 50:25-49

30. Williams AG, Coleman GS (1992) In: Wright ADG (ed) The rumen protozoa. Springer, New York, pp 4-83

31. Williams AG, Coleman GS (1997) The rumen protozoa. In: Hobson PN, Stewart CS (eds) The rumen microbial ecosystem. Blackie Academic \& Professional, New York

32. Wolin MJ (1974) Metabolic interactions among intestinal microorganisms. Am J Clin Nutr 27:1320-1328 